\title{
A New Lifetime Distribution: The Beta Modified Weibull Power Series Distribution
}

\author{
Narges Yarmoghaddam and Ehsan Bahrami Samani* \\ Shahid Beheshti University
}

Received: 5/23/2019 Approved: 3/12/2020

\begin{abstract}
In this paper, we propose a new parametric distribution which called as the Beta Modified Weibull Power Series (BMWPS) distribution. This distribution is obtained by compounding Beta Modified Weibull (BMW) and power series distributions. BMWPS distribution contains, as special submodels, such as Beta Modified Weibull Poisson (BMWP) distribution, Beta Modified Weibull Geometric (BMWG) distribution, Beta Modified Weibull Logarithmic (BMWL) distribution, among others. We obtain closed-form expressions for the cumulative distribution, density, survival function, failure rate function, the r-th raw moment and the moments of order statistics. A full likelihood-based approach that allows yielding maximum likelihood estimates of the BMWPS parameters is used. Finally, application to the Aarset data are given.
\end{abstract}

Keywords. Lifetime; Beta Modified Weibull distribution; power series distribution; maximum Likelihood estimation; hazard function; Fisher's information matrix.

MSC 2010: 62F10, 62N05.

\footnotetext{
* Corresponding author
} 


\section{Introduction}

The Weibull distribution, having exponential and Rayleigh as special cases, is a very popular distribution for modeling lifetime data and for modeling phenomenon with monotone failure rates. When modeling monotone hazard rates, the Weibull distribution may be an initial choice because of its negatively and positively skewed density shapes. However, it does not provide a reasonable parametric fit for modeling phenomenon with non-monotone failure rates such as the bathtub shaped and the unimodal failure rates which are common in reliability and biological studies. Such bathtub hazard curves have nearly flat middle portions and the corresponding densities have a positive anti-mode. The last few years, new classes of distributions were proposed based on modifications of the Weibull distribution to cope with bathtub shaped failure rate. A good review of some of these distributions are presented in Pham and Lai (2007). Between these, the exponentiated Weibull (EW) distribution introduced by Mudholkar et al. (1995, 1996), the additive Weibull distribution (Xie and Lai, 1995), the extended Weibull distribution presented by Xie et al. (2002), the modified Weibull (MW) distribution proposed by Lai et al. (2003), the beta exponential (BE) distribution by Nadarajah and Kotz (2005), the beta Weibull (BW) distribution studied by Cordeiro et al.(2008), the extended flexible Weibull distribution by Bebbington et al. (2007) and the generalized modified Weibull (GMW) by Carrasco et al. (2008). Silva et al. (2010) presented generalized modified Weibull (BMW) distribution.

Several distributions have been proposed in the literature to model lifetime data by compounding some useful lifetime distributions. For example, Kus (2007) introduced exponential-Poisson (EP) distribution, exponentialpower series (EPS) by Chahkandi and Ganjali (2009); Weibull-power series (WPS) by and Morais and Barreto-Souza (2011); exponentiated exponentialPoisson (EEP) by and Barreto-Souza and Cribari-Neto (2009); complementary Weibull geometric by Tojeiro et al. (2014); the Lomaxlogarithmic by Al-Zahrani and Sagor (2014); Weibull Rayleigh by Faton and Elbatal (2015); the Burr XII negative Binomial by Ramos et al. (2015); the compound class of linear failure rate-power series by Mahmoudi and Jafari (2014). Bagheri et al. (2016) presented the generalized modified Weibull power series (GMWPS) distribution. This distribution is constructed based on a latent complementary risk problem and is obtained by compounding generalized modified Weibull (GMW) and power series distributions. 
In this paper, the main purpose of the modification and extension forms of the beta Weibull distribution is to describe. We introduce a new distribution with six parameters, so called the beta modified Weibull (BMW) distribution. Overall, it is hoped that current research can be useful in providing information for the theoretical study and application of Beta modified Weibull (BMW) related distributions.

The rest of the paper is organized as follows. In Section 2, we explain about our proposed distribution and in Section 3, we propose the new class of Beta modified Weibull power series (BMWPS) distributions, we give the density and failure rate functions of the BMWPS distribution. Furthermore, we present some special distributions which are studied in detail, and we show that the failure rate can be increasing or decreasing, it can also be bathtub-shaped and unimodal. Additionally, we derive quantiles and moments of BMWPS distributions, and the probability density function of the ith order statistic. Expressions for the $k$ th raw moments of the BMWPS distribution are given in Section 4. We discuss estimation by maximum likelihood and provide an expression for Fishers information matrix in Section 5. In Section 6, we consider simulation study for the Beta Modified Weibull Poisson (BMWP) distribution. Applications to a real data set is given to show the flexibility and potentiality of the new distribution in Section 7 . Finally Section 8 concludes the paper.

\section{Motivation}

We present the Beta Modified Weibull Power Series distribution, which is extended than previous distributions (via Bagheri et al., 2016). In Table 1, we present the Beta Modified Weibull distributions and sub distributions. Also, in Figure 1, we show relationships between the Beta Modified Weibull Power Series distribution families. So, we propose and extent a new parametric distribution which called as the Beta Modified Weibull Power Series (BMWPS) distribution.

\section{The Beta Modified Weibull Power Series (BMWPS) distribution}

The four-parameter distribution known as generalized modified Weibull (BMW) distribution, was introduced by Silva et al. (2010). The cumulative distri- 
Table 1. The class of Beta Modified Weibull distributions .

Power Series : Poisson, Geometric, Logarithmic, Binomial

\begin{tabular}{|c|c|c|c|c|c|}
\hline Distribution & $a$ & $b$ & $\lambda$ & $\gamma$ & $\alpha$ \\
\hline BMW & & & & & The Beta Modified Weibull Distribution \\
\hline BMR & & & & 2 & The Beta Modified Rayleigh Distribution \\
\hline BME & & & & 1 & The Beta Modified Exponential Distribution \\
\hline GMW & & 1 & & & The Generalized Modified Weibull Distribution \\
\hline GMR & & 1 & & 2 & The Generalized Modified Rayleigh Distribution \\
\hline GME & & 1 & & 1 & The Generalized Modified Exponential Distribution \\
\hline BW & & & 0 & & The Beta Weibull Distribution \\
\hline $\mathrm{BR}$ & & & 0 & 2 & The Beta Rayleigh Distribution \\
\hline $\mathrm{BE}$ & & & 0 & 1 & The Beta Exponential Distribution \\
\hline MW & 1 & 1 & & & The Modified Weibull Distribution \\
\hline MR & 1 & 1 & & 2 & The Modified Rayleigh Distribution \\
\hline ME & 1 & 1 & & 1 & The Modified Exponential Distribution \\
\hline GW & & 1 & 0 & & The Generalized Weibull Distribution \\
\hline GR & & 1 & 0 & 2 & The Generalized Rayleigh Distribution \\
\hline GE & & 1 & 0 & 1 & The Generalized Exponential Distribution \\
\hline $\mathrm{W}$ & 1 & 1 & 0 & & The Weibull Distribution \\
\hline $\mathrm{R}$ & 1 & 1 & 0 & 2 & The Rayleigh Distribution \\
\hline $\mathrm{E}$ & 1 & 1 & 0 & 1 & The Exponential Distribution \\
\hline
\end{tabular}

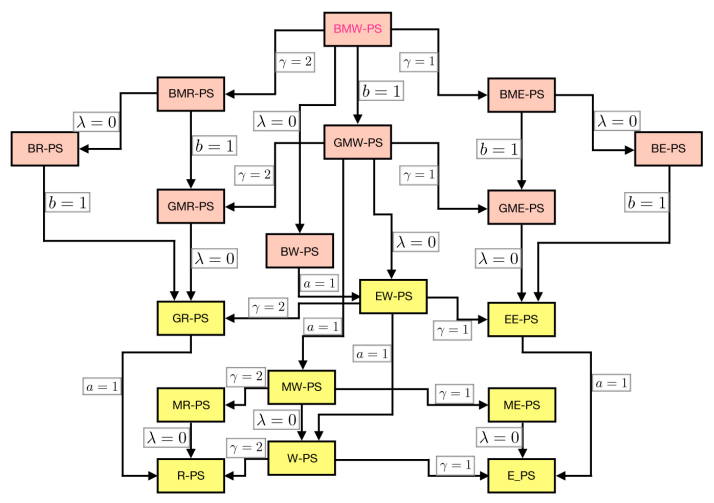

Figure 1. Relationships between the class of Beta Modified Weibull Power Series distribution. 
Table 2. Useful quantities of some power series distributions. Note that $\theta \in(0, \infty)$ for the Poisson distribution and $\theta \in(0,1)$ for all other distributions.

\begin{tabular}{cccccc}
\hline \hline Distribution & $a_{n}$ & $C(\theta)$ & $C^{\prime}(\theta)$ & $C^{\prime \prime}(\theta)$ & $C(\theta)^{-1}$ \\
\hline Poisson & $n !^{-1}$ & $e^{\theta}-1$ & $e^{\theta}$ & $e^{\theta}$ & $\log (\theta+1)$ \\
Logarithmic & $n^{-1}$ & $-\log (1-\theta)$ & $(1-\theta)^{-1}$ & $(1-\theta)^{-2}$ & $1-e^{-\theta}$ \\
Geometric & 1 & $\theta(1-\theta)^{-1}$ & $(1-\theta)^{-2}$ & $2(1-\theta)^{-3}$ & $\theta(\theta+1)^{-1}$ \\
Binomial & $\left(\begin{array}{c}m \\
n\end{array}\right)$ & $(\theta+1)^{m}-1$ & $m(\theta+1)^{m-1}$ & $\frac{m(m-1)}{(\theta+1)^{2-m}}$ & $(\theta-1)^{\frac{1}{m}}-1$ \\
\hline
\end{tabular}

bution function (cdf) of the BMW distribution with six parameters $a>0$, $b>0, \sigma>0, k>0$ and $\alpha, \beta \geqslant 0$ such that $\alpha+\beta>0$ is given by

$$
G(y)=\left(\frac{1}{B(a, b)} \int_{0}^{1-e^{-\alpha y-\beta y^{\sigma}}} t^{a-1}(1-t)^{b-1} d t\right)^{k}
$$

and the density function of the BMW distribution is given by

$$
\begin{aligned}
g(y) & =k\left(\alpha+\beta \sigma y^{\sigma-1}\right) e^{-\alpha y-\beta y^{\sigma}}\left(\frac{1}{b(a, b)}\right)^{k}\left(1-e^{-\alpha y-\beta y^{\sigma}}\right)^{a-1}\left(e^{-\alpha y-\beta y^{\sigma}}\right)^{b-1} \\
& \times\left(\int_{0}^{1-e^{-\alpha y-\beta y^{\sigma}}} t^{a-1}(1-t)^{b-1} d t\right)^{k-1}
\end{aligned}
$$

The importance of this distribution lies in its ability to model monotone as well as non-monotone failure rates, which are quite common in lifetime problems and reliability. Let $N$ be a random variable denoting the number of failure causes, $N=1,2, \ldots$ and considering $N$ following a power series distribution (truncated at zero) with probability function given by

$$
p(N=n)=\frac{a_{n} \theta^{n}}{C(\theta)}, \quad n=1,2, \ldots \text { and } \theta \in(0, S),
$$

where $a_{1}, a_{2}, \ldots$ is a sequence of nonnegative real numbers, where at least one of them is strictly positive, $S$ is a positive number no greater than the ratio of convergence of the power series $\sum_{n=1}^{\infty} a_{n} \theta^{n}$ and $C(\theta)=\sum_{n=1}^{\infty} a_{n} \theta^{n}$. Useful quantities of some power series distributions are given in Table 2 .

The Beta Modified Weibull Power Series Distribution, denoted by BMWPS $(\alpha, \beta, \sigma, a, b, k, \theta)$, is defined by the marginal cdf of $X=\max \left\{Y_{1}, Y_{2}, \ldots, Y_{N}\right\}$, 
i.e.,

$$
F(x)=1-\frac{C\left(\theta\left(1-\left(I_{1-e^{-\alpha x-\beta x^{\sigma}}}(a, b)\right)^{k}\right)\right)}{C(\theta)}
$$

where $a, b, \sigma, k, \theta>0$ and $\alpha, \beta \geqslant 0$, and also

$$
I_{1-e^{-\alpha x-\beta x^{\sigma}}}(a, b)=\frac{1}{B(a, b)} \int_{0}^{1-e^{-\alpha x-\beta x^{\sigma}}} t^{a-1}(1-t)^{b-1} d t .
$$

The pdf of the $\operatorname{BMWPS}(\alpha, \beta, \sigma, a, b, k, \theta)$ is given by

$$
\begin{aligned}
& f(x)=k \theta \frac{1}{B(a, b)}\left(1-e^{-\alpha x-\beta x^{\sigma}}\right)^{a-1}\left(e^{-\alpha x-\beta x^{\sigma}}\right)^{b-1}\left(\alpha+\beta \sigma x^{\sigma-1}\right) e^{-\alpha x-\beta x^{\sigma}} \\
& \times \frac{\left(I_{1-e^{-\alpha x-\beta x^{\sigma}}}(a, b)\right)^{k-1} C^{\prime}\left(\theta\left(1-\left(I_{1-e^{-\alpha x-\beta x^{\sigma}}}(a, b)\right)^{k}\right)\right)}{C(\theta)}
\end{aligned}
$$

The survival function and hazard rate function of the BMWPS distribution are given, respectively, by

$$
s(x)=\frac{C\left(\theta\left(1-\left(I_{1-e^{-\alpha y-\beta y^{\sigma}}}(a, b)\right)^{k}\right)\right)}{C(\theta)},
$$

and

$$
\begin{aligned}
h(x) & =k \theta \frac{1}{B(a, b)}\left(1-e^{-\alpha x-\beta x^{\sigma}}\right)^{a-1}\left(e^{-\alpha x-\beta x^{\sigma}}\right)^{b-1}\left(\alpha+\beta \sigma x^{\sigma-1}\right) e^{-\alpha x-\beta x^{\sigma}} \\
& \times\left(I_{1-e^{-\alpha x-\beta x^{\sigma}}}(a, b)\right)^{k-1} \times \frac{C^{\prime}\left(\theta\left(1-\left(I_{1-e^{-\alpha x-\beta x^{\sigma}}}(a, b)\right)^{k}\right)\right)}{C\left(\theta\left(1-\left(I_{1-e^{-\alpha x-\beta x^{\sigma}}}(a, b)\right)^{k}\right)\right)}
\end{aligned}
$$

\section{Special Cases}

In the following subsection we studied in details some special cases of the class BMWPS of distributions. This class of distribution contains several lifetime models such as: Beta modified Weibull Poisson (BMWP), Beta modified Weibull logarithmic (BMWL) and Beta modified Weibull geometric (BMWG) distributions as special cases. 


\subsection{Beta Modified Weibull Poisson Distribution}

The Beta modified Weibull Poisson (BMWP) distribution is a special case of BMWPS distributions with $a_{n}=\frac{1}{n !}$ and $C(\theta)=e^{\theta}-1$. Using cdf (4), the cdf of Beta modified Weibull poisson (BMWP) distribution is given by

$$
F_{B M W P}(x)=1-\frac{e^{\theta\left(1-\left(I_{\left.\left.1-e^{-\alpha x-\beta x^{\sigma}}(a, b)\right)^{k}\right)}-1\right.\right.}}{e^{\theta}-1}
$$

where $a, b, \sigma, k, \theta>0$ and $\alpha, \beta \geqslant 0$. The associated pdf and hazard rate function of this distribution are given, respectively, by

$$
\begin{aligned}
f_{B M W P}(x) & =k \theta \frac{1}{B(a, b)}\left(1-e^{-\alpha x-\beta x^{\sigma}}\right)^{a-1}\left(e^{-\alpha x-\beta x^{\sigma}}\right)^{b-1}\left(\alpha+\beta \sigma x^{\sigma-1}\right) \\
& \times \frac{e^{-\alpha x-\beta x^{\sigma}}\left(I_{\left.1-e^{-\alpha x-\beta x^{\sigma}}(a, b)\right)^{k-1}} e^{\theta\left(1-\left(I_{\left.1-e^{-\alpha x-\beta x^{\sigma}}(a, b)\right)^{k}}\right)\right.}\right.}{e^{\theta}-1}
\end{aligned}
$$

and

$$
\begin{aligned}
h_{B M W P}(x) & =k \theta \frac{1}{B(a, b)}\left(1-e^{-\alpha x-\beta x^{\sigma}}\right)^{a-1}\left(e^{-\alpha x-\beta x^{\sigma}}\right)^{b-1}\left(\alpha+\beta \sigma x^{\sigma-1}\right) \\
& \times \frac{e^{-\alpha x-\beta x^{\sigma}}\left(I_{\left.1-e^{-\alpha x-\beta x^{\sigma}}(a, b)\right)^{k-1}} e^{\theta\left(1-\left(I_{\left.1-e^{-\alpha x-\beta x^{\sigma}}(a, b)\right)^{k}}\right)\right.}\right.}{e^{\theta\left(1-\left(I_{\left.1-e^{-\alpha x-\beta x^{\sigma}}(a, b)\right)^{k}}\right)\right.}-1}
\end{aligned}
$$

The plots of pdf, cdf and hazard rate function of BMWP distribution for some values of $\alpha, \beta, \sigma, a, b, k$ and $\theta$ are given in Figure 2.

The new distribution that presents bathtub-shaped failure rate are very useful in survival analysis. The modeling and analysis of lifetimes is an important aspect of statistical work in a wide variety of scientific and technological fields. The new distribution due to its flexibility in accommodating all the forms of the risk function seems to be an important distribution that can be used in a variety of problems in modeling survival data. 

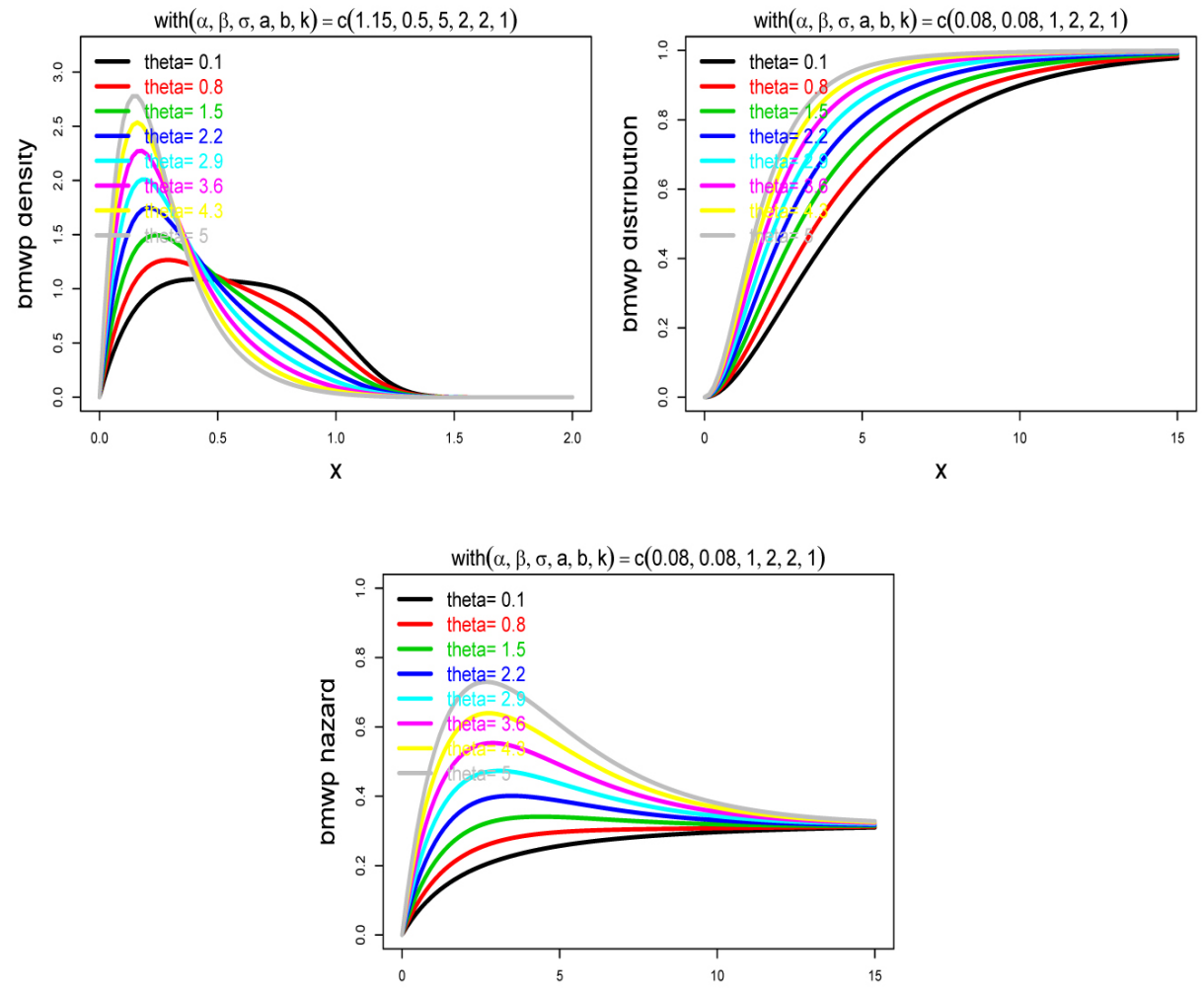

Figure 2. Plots of density function, cumulative distribution function and survival function of BMWP distribution for different values of the vector $(\alpha, \beta, \sigma, a, b, k, \theta)$. 


\subsection{Beta Modified Weibull Geometric Distribution}

The Beta modified Weibull Geometric (BMWG) distribution is a special case of BMWPS distributions with $a_{n}=1$ and $C(\theta)=\theta(1-\theta)^{-1}$. Using cdf (4), the cdf of Beta modified Weibull geometric (BMWG) distribution is given by

$$
F_{B M W G}(x)=\frac{\left(I_{1-e^{-\alpha x-\beta x^{\sigma}}}(a, b)\right)^{k}}{1-\theta\left(1-\left(I_{1-e^{-\alpha x-\beta x^{\sigma}}}(a, b)\right)^{k}\right)}
$$

where $a, b, \sigma, k, \theta>0$ and $\alpha, \beta \geqslant 0$. The pdf is

$$
\begin{aligned}
f_{B M W G}(x) & =k \theta \frac{1}{B(a, b)}\left(1-e^{-\alpha x-\beta x^{\sigma}}\right)^{a-1}\left(e^{-\alpha x-\beta x^{\sigma}}\right)^{b-1} \\
& \times\left(\alpha+\beta \sigma x^{\sigma-1}\right) e^{-\alpha x-\beta x^{\sigma}}\left(I_{1-e^{-\alpha x-\beta x^{\sigma}}}(a, b)\right)^{k-1} \\
& \times \frac{\left(1-\theta\left(1-\left(I_{\left.\left.\left.1-e^{-\alpha x-\beta x^{\sigma}}(a, b)\right)^{k}\right)\right)^{-2}}\right.\right.\right.}{\theta(1-\theta)^{-1}} .
\end{aligned}
$$

The plots of pdf, cdf and hazard rate function of BMWG distribution for some values of $\alpha, \beta, \sigma, a, b, k$ and $\theta$ are given in Figure 3.

The hazard rate function of BMWG distribution is given by

$$
\begin{aligned}
h_{G M W G}(x) & =A\left(1-\theta\left(1-\left(I_{1-e^{-\alpha x-\beta x^{\sigma}}}(a, b)\right)^{k}\right)\right)^{-2} \\
& \times \frac{\left(1-\theta\left(1-\left(I_{1-e^{-\alpha x-\beta x^{\sigma}}}(a, b)\right)^{k}\right)\right)}{\theta\left(1-\left(1-\left(I_{1-e^{-\alpha x-\beta x^{\sigma}}}(a, b)\right)^{k}\right)(1-\theta)\right)}
\end{aligned}
$$

where

$$
\begin{aligned}
A & =k \theta \frac{1}{B(a, b)}\left(1-e^{-\alpha x-\beta x^{\sigma}}\right)^{a-1}\left(e^{-\alpha x-\beta x^{\sigma}}\right)^{b-1}\left(\alpha+\beta \sigma x^{\sigma-1}\right) \\
& \times e^{-\alpha x-\beta x^{\sigma}}\left(I_{1-e^{-\alpha x-\beta x^{\sigma}}}(a, b)\right)^{k-1} .
\end{aligned}
$$

\subsection{Beta Modified Weibull Logarithmic Distribution}

The Beta modified Weibull logarithmic (BMWL) distribution is a special case of BMWPS distributions with $a_{n}=\frac{1}{n}$ and $C(\theta)=-\log (1-\theta)$. Using cdf 

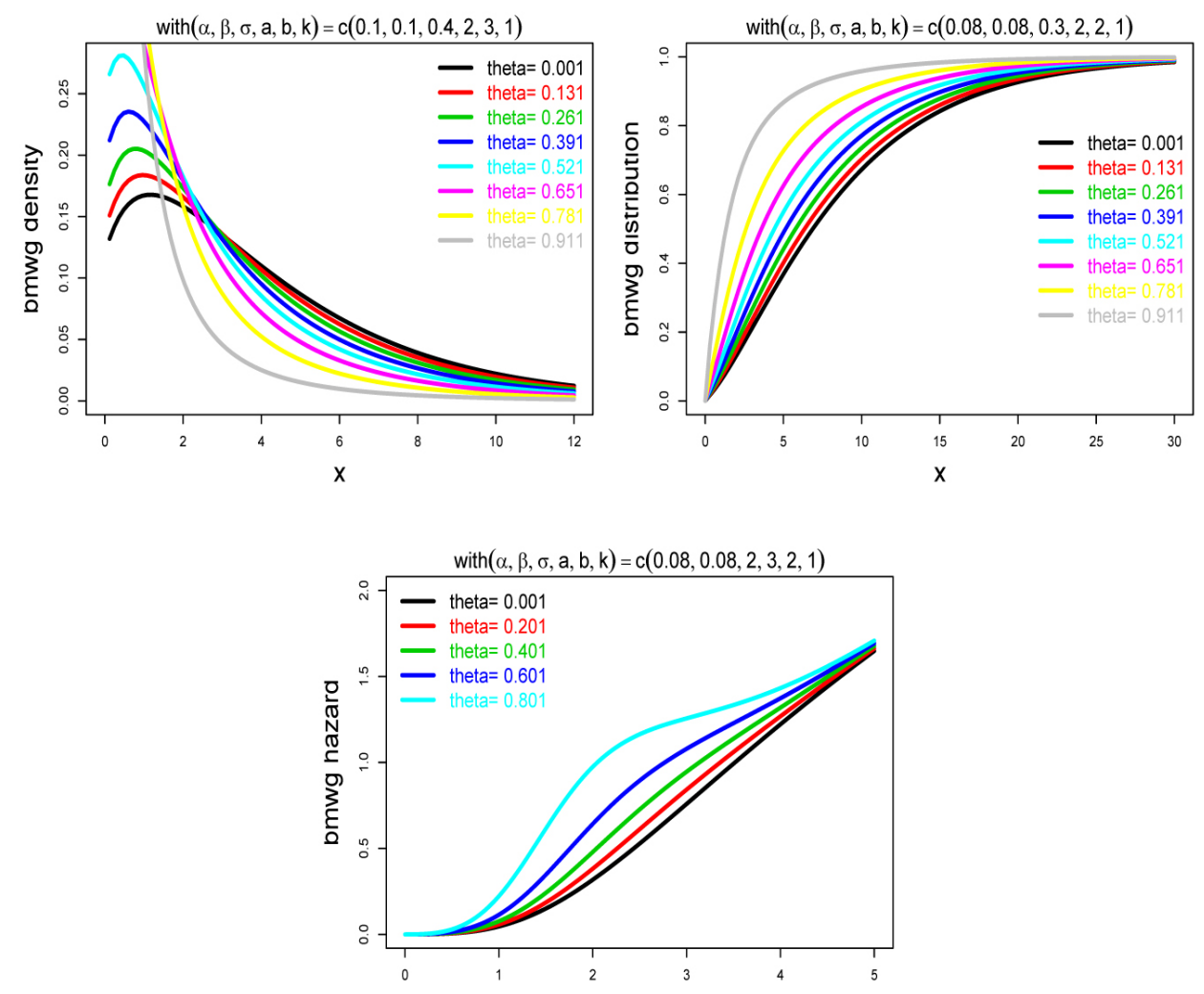

Figure 3. Plots of density function, cumulative distribution function and hazard rate function of BMWG distribution for different values of the vector $(\alpha, \beta, \sigma, a, b, k, \theta)$. 
(4), the cdf of generalized modified Weibull logarithmic (BMWl) distribution is given by

$$
F_{B M W L}(x)=1-\frac{\log \left(1-\theta\left(1-\left(I_{1-e^{-\alpha x-\beta x^{\sigma}}}(a, b)\right)^{k}\right)\right)}{\log (1-\theta)}
$$

where $a, b, \sigma, k, \theta>0$ and $\alpha, \beta \geqslant 0$. The plots of pdf, cdf and hazard rate function of BMWL distribution for some values of $\alpha, \beta, \sigma, a, b, k$ and $\theta$ are given in Figure 4.
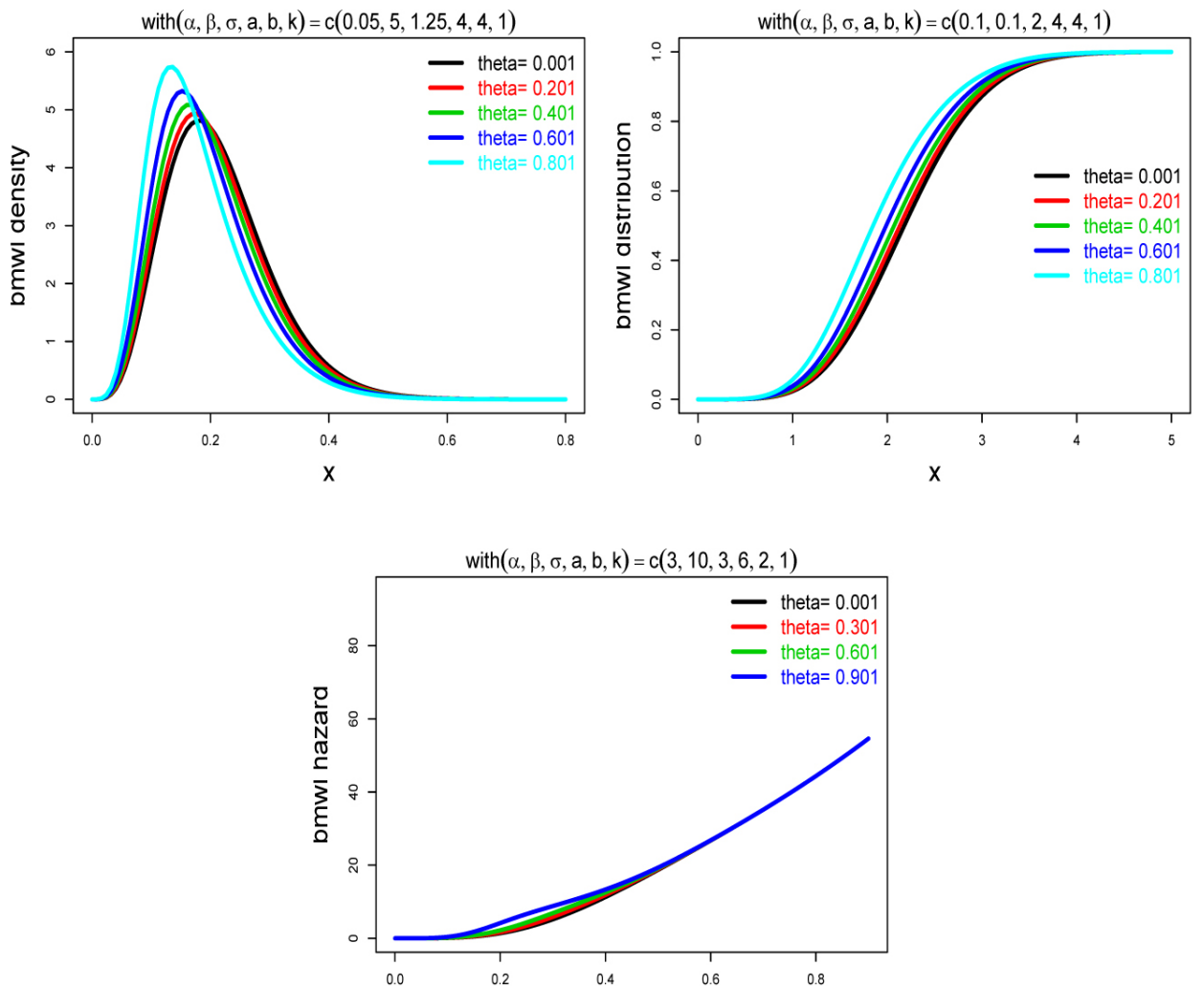

Figure 4. Plots of density function, cumulative distribution function and hazard rate function of BMWL distribution for different values of the vector $(\alpha, \beta, \sigma, a, b, k, \theta)$. 
The associated pdf and hazard rate functions are given, respectively, by

$$
\begin{aligned}
f_{B M W L}(x) & =-k \theta \frac{1}{B(a, b)}\left(1-e^{-\alpha x-\beta x^{\sigma}}\right)^{a-1}\left(e^{-\alpha x-\beta x^{\sigma}}\right)^{b-1}\left(\alpha+\beta \sigma x^{\sigma-1}\right) \\
& \times e^{-\alpha x-\beta x^{\sigma}}\left(I_{\left.1-e^{-\alpha x-\beta x^{\sigma}}(a, b)\right)^{k-1}}\right. \\
& \times \frac{\left(1-\theta\left(1-\left(I_{\left.\left.\left.1-e^{-\alpha x-\beta x^{\sigma}}(a, b)\right)^{k}\right)\right)^{-1}}\right.\right.\right.}{\log (1-\theta)}
\end{aligned}
$$

and

$$
\begin{aligned}
h_{B M W L}(x) & =-k \theta \frac{1}{B(a, b)}\left(1-e^{-\alpha x-\beta x^{\sigma}}\right)^{a-1}\left(e^{-\alpha x-\beta x^{\sigma}}\right)^{b-1}\left(\alpha+\beta \sigma x^{\sigma-1}\right) \\
& \times e^{-\alpha x-\beta x^{\sigma}}\left(I_{\left.1-e^{-\alpha x-\beta x^{\sigma}}(a, b)\right)^{k-1}}\right. \\
& \times \frac{\left(1-\theta\left(1-\left(I_{\left.\left.\left.1-e^{-\alpha x-\beta x^{\sigma}}(a, b)\right)^{k}\right)\right)^{-1}}\right.\right.\right.}{\log \left(1-\theta\left(1-\left(I_{1-e^{-\alpha x-\beta x^{\sigma}}}(a, b)\right)^{k}\right)\right)} .
\end{aligned}
$$

\section{Statistical Properties}

In this section, we proposed some of the basic statistical properties of the BMWP. For examples, we provide quantiles and order statistic, Renyi and Shannon entropies Moments. The moment generating function, Residual life function, Probability weighted moments, Mean deviations, Bonferroni and Lorenz curves are also provided for the BMWP. The BMWPS distribution is a mixture of the BMW distribution with weights defined by the power series distribution.

\section{Quantiles, Moments and Order Statistics}

The quantiles of a distribution can be used in data generation from a distribution. The quantile $x_{q}$ of the $\operatorname{BMWPS}(\alpha, \beta, \sigma, a, b, k, \theta)$ is the real solution of the following equation:

$$
\left(I_{1-e^{-\alpha x_{q}-\beta x_{q}^{\sigma}}}(a, b)\right)-\left(1-\frac{C^{-1}((1-q) C(\theta))}{\theta}\right)^{\frac{1}{k}}=0 .
$$

The above equation has no closed form solution in $x_{q}$, so we have to use a numerical technique such as a Newton-Raphson method to get the quantile. 
The pdf $f_{i: n}$ of the $i$ th order statistic for a random sample $X_{1}, X_{2}, \ldots, X_{n}$ from the BMWPS distribution is given by

$$
\begin{aligned}
f_{i: n}(x) & =\frac{1}{B(i, n-i+1)} f(x) F(x)^{i-1}[1-F(x)]^{n-i} \\
& =\frac{1}{B(i, n-i+1)} f(x) \sum_{j=0}^{n-i}\left(\begin{array}{c}
n-i \\
j
\end{array}\right)(-1)^{j} \\
& \times\left[\frac{C\left(\theta\left(1-\left(I_{\left.1-e^{-\alpha x-\beta x^{\sigma}}(a, b)\right)^{k}}\right)\right)\right.}{C(\theta)}\right]^{j+i-1}
\end{aligned}
$$

and the cdf is

$$
\begin{aligned}
F_{i: n}(x) & =\sum_{k=i}^{n}\left(\begin{array}{l}
n \\
k
\end{array}\right) F(x)^{k}[1-F(x)]^{n-k} \\
& =\sum_{k=i}^{n} \sum_{j=0}^{n-k}\left(\begin{array}{c}
n-k \\
j
\end{array}\right)\left(\begin{array}{l}
n \\
k
\end{array}\right)(-1)^{j}\left[\frac{C\left(\theta\left(1-\left(I_{1-e^{-\alpha x-\beta x^{\sigma}}}(a, b)\right)^{k}\right)\right)}{C(\theta)}\right]^{j+k} .
\end{aligned}
$$

Carrasco et al. (2008, Sect. 4) obtained an infinite representation for the $r$ th moment of the $B M W(\alpha, \beta, \sigma, a, b, k)$ distribution. If $\mathrm{Y}$ has the $B M W(\alpha, \beta, \sigma, a, b, k)$, the $r$ th moment of Y say $\mu_{r}^{\prime}$, is given as follows

$$
\mu_{r}^{\prime}=\sum_{j=0}^{\infty} \sum_{i_{1}, \ldots, i_{r}=1}^{\infty} w_{j} \frac{A_{i_{1}, \ldots, i_{r}} \Gamma\left(S_{r} / \sigma+1\right)}{(\alpha(b+j))^{\frac{S_{r}}{\sigma}}}
$$

where $A_{i_{1}, \ldots, i_{r}}=a_{i_{1}} \ldots a_{i_{r}}, S_{r}=i_{1}+\ldots+i_{r}$,

$$
a_{i}=\frac{(-1)^{i+1} i^{i-2} \lambda^{i-1}}{(i-1) ! \sigma^{i-1}}
$$

and

$$
w_{j}=w_{j}(a, b)=\frac{(-1)^{j} \Gamma(a)}{B(a, b) \Gamma(a-j)(b+j) j !} .
$$

Let that $Y_{i: n}$ be the $i$ th order statistic of a random sample from the BMW distri- 
bution. The $r$ th moment of $\operatorname{BMWPS}(\alpha, \beta, \sigma, a, b, k, \theta)$, is given as follows

$$
\begin{aligned}
\mu_{r}=E\left(X^{r}\right) & =\sum_{i=1}^{\infty} P(N=n) E\left(Y_{(n)}^{k}\right) \\
& =\sum_{n=1}^{\infty} \frac{a_{n} \theta^{n}}{C(\theta)} n \sum_{j=0}^{\infty} \frac{(-1)^{j} \Gamma(a)}{B(a, b) \Gamma(a-j)(b+j) j !} \\
& \times \sum_{i_{1}, \ldots, i_{r}=1}^{\infty} \frac{A_{i_{1}, \ldots, i_{r}} \Gamma\left(S_{r} / \sigma+1\right)}{(\alpha(b+j))^{\frac{S_{r}}{\sigma}}}
\end{aligned}
$$

Based on the results given in (17), the measures of skewness and kurtosis of the $\operatorname{BMWP}(\alpha, \beta, \sigma, a, b, k, \theta)$ can be obtained according to the following relations, respectively,

$$
\begin{gathered}
\text { skewness }=\frac{\mu_{3}-3 \mu_{1} \mu_{2}+2 \mu_{1}^{3}}{\left(\mu_{2}-\mu_{1}\right)^{\frac{3}{2}}}, \\
\text { kurtosis }=\frac{\mu_{4}-4 \mu_{3} \mu_{1}+6 \mu_{1}{ }^{2} \mu_{2}-3 \mu_{1}^{4}}{\left(\mu_{2}-\mu_{1}\right)^{2}} .
\end{gathered}
$$

To illustrate the behavior of the skewness and kurtosis when $\theta$ varies, Figure 5 presents the GaltonÂ s skewness (Johnson et al., 1994, p. 40) and Moors Â kurtosis (Moors, 1988) as functions of $\theta$ for selected values of $\alpha, \beta$ and $\sigma$.

\section{Moment Generating Function}

If $\mathrm{Y}$ has the $\operatorname{BMW}(\alpha, \beta, \sigma, a, b, k)$, with the moment generating function (mgf) say $M_{Y}(t)$, then the moment generating function (mgf) of $\operatorname{BMWPS}(\alpha, \beta, \sigma, a, b, k, \theta)$, is given as follows

$$
\begin{aligned}
M_{x}(t) & =\sum_{r=0}^{\infty} \frac{t^{r}}{r !} E\left(x^{r}\right) \\
& =\sum_{r=0}^{\infty} \sum_{n=1}^{\infty} \frac{t^{r}}{r !} \frac{a_{n} \theta^{n}}{C(\theta)} n \sum_{j=0}^{\infty} \frac{(-1)^{j} \Gamma(a)}{B(a, b) \Gamma(a-j)(b+j) j !} \\
& \times \sum_{i_{1}, \ldots, i_{r}=1}^{\infty} \frac{A_{i_{1}, \ldots, i_{r}} \Gamma\left(\frac{S_{r}}{\sigma}+1\right)}{(\alpha(j+1))^{\frac{S_{r}}{\sigma}}}
\end{aligned}
$$


Galton's skewness

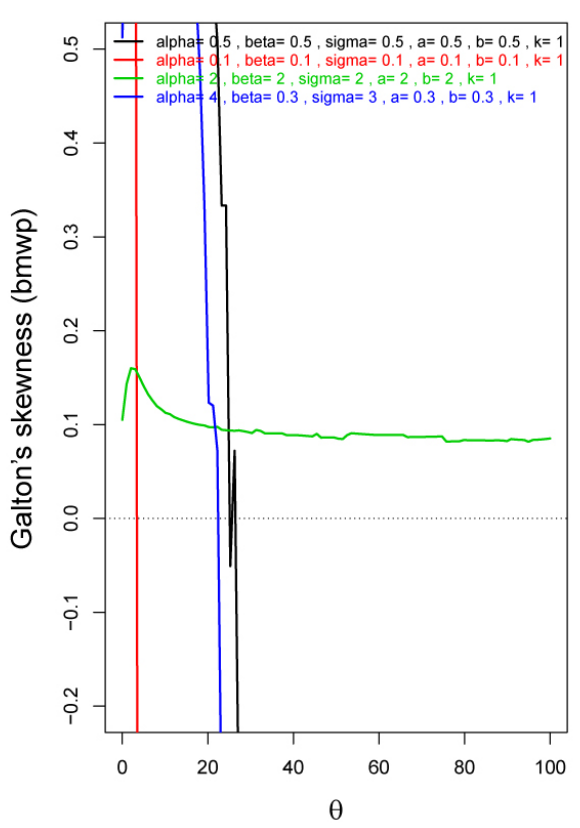

Moor's kurtosis

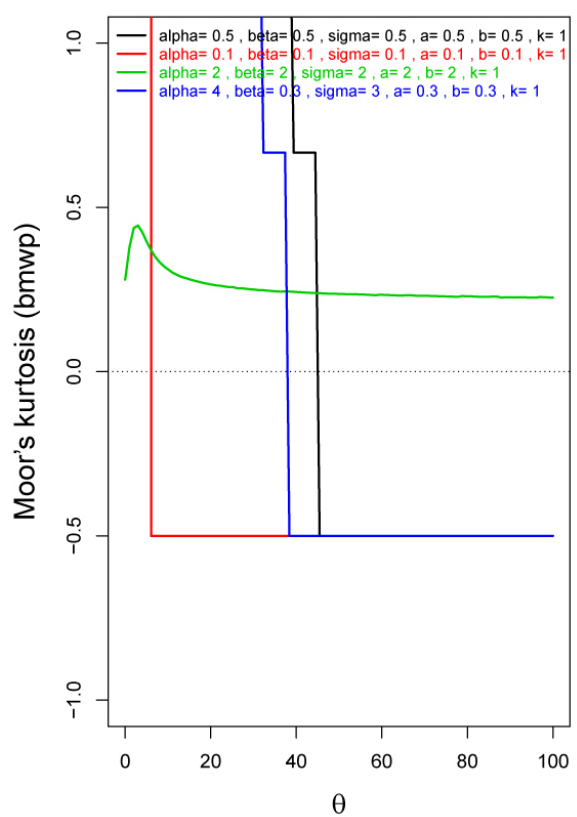

Figure 5. The effect of $\theta$ on the GaltonÂs skewness and Moors $\hat{A}$ kurtosis for different values of $\alpha, \beta$ and $\sigma$. 


\section{Mean Deviations}

The amount of scatter in a population can be measured by the totality of deviations from the mean and median. The mean deviation from the mean is a robust statistic, being more resilient to outliers in a data set than the standard deviation. For a random variable $X$ with pdf $f(x)$, cdf $F(x)$, mean $\mu$ and median $M$, the mean deviation from the mean and the mean deviation from the median are defined by

$$
\delta_{1}(x)=\int_{0}^{\infty}|x-\mu| f(x) d x=2 \mu F(\mu)-2 I(\mu)
$$

and

$$
\delta_{2}(x)=\int_{0}^{\infty}|x-M| f(x) d x=2 M F(M)-M+\mu-2 I(M)
$$

respectively, where

$$
I(a)=\int_{0}^{a} x f(x) d x=\frac{\theta}{C(\theta)} \sum_{n=1}^{\infty} \theta^{n-1} a_{n} \varpi^{a}{ }_{1}\{\alpha, \beta, \sigma, a, b, n k\} .
$$

\section{Bonferroni and Lorenz Curves}

Study of income inequality has gained a lot of importance over the last many years. Lorenz curve and the associated Gini index are undoubtedly the most popular indices of income inequality. For a random variable $\mathrm{X}$ with cdf $\mathrm{F}($.$) , the Bonferroni curve$ is given by

$$
\begin{aligned}
& B_{F}[F(x)]=\frac{1}{\mu F(x)} \int_{0}^{x} u f(u) d u=\frac{\theta}{\mu\left(C(\theta)-C\left(\theta\left(1-\left(I_{1-e^{-\alpha x-\beta x^{\sigma}}}(a, b)\right)^{k}\right)\right)\right)} \\
& \times \sum_{n=1}^{\infty} \theta^{n-1} a_{n} \varpi^{a}{ }_{1}\{\alpha, \beta, \sigma, a, b, n k\} .
\end{aligned}
$$

The Lorenz curve of the BMWPS distribution can be obtained via the expression

$$
\begin{aligned}
L_{F}[F(x)] & =B_{F}[F(x)] F(x)=\frac{1}{\mu} \int_{0}^{x} u f(u) d u \\
& =\frac{\theta}{\mu C(\theta)} \sum_{n=1}^{\infty} \theta^{n-1} a_{n} \varpi^{a}{ }_{1}\{\alpha, \beta, \sigma, a, b, n k\}
\end{aligned}
$$

\section{Estimation and Inference}

Standard statistical techniques such as method of maximum likelihood can always be used for parametric estimation. The likelihood equations, given the complete or 
censored failure data set, can be derived and solved. Parameter estimation is usually a difficult problem even for a five parameter BMWPS distribution. Methods like the maximum likelihood estimation will not yield a closed form solution. Different methods can be used to estimate the model parameters. Among these methods, the Maximum Likelihood Estimation method is the most commonly used method for model estimation. In this subsection, we use the maximum likelihood procedure to derive the point and interval estimates of the parameters.

$$
\begin{aligned}
L= & n \ln (\theta)-n \ln \left(C^{\prime}(\theta)\right)+n \ln (k)+k n \ln \left(\frac{1}{B(a, b)}\right) \\
& +(a-1) \sum_{i=1}^{n} \ln \left(1-e^{-\alpha x_{i}-\beta x_{i}^{\sigma}}\right)+(b-1) \sum_{i=1}^{n} \ln \left(e^{-\alpha x_{i}-\beta x_{i}^{\sigma}}\right) \\
& +\sum_{i=1}^{n} \ln \left(\alpha+\beta \sigma x_{i}^{\sigma-1}\right) \sum_{i=1}^{n}\left(-\alpha x_{i}-\beta x_{i}^{\gamma}\right) \\
& +(k-1) \sum_{i=1}^{n} \ln \left(\int_{0}^{1-e^{-\alpha x_{i}-\beta x_{i}^{\sigma}}} t^{a-1}(1-t)^{b-1} d t\right) \\
& +\sum_{i=1}^{n} \ln \left(C ^ { \prime } \left(\theta \left(1-\left(I_{\left.\left.\left.\left.1-e^{-\alpha x_{i}-\beta x_{i}^{\sigma}}(a, b)\right)^{k}\right)\right)\right) .}\right.\right.\right.\right.
\end{aligned}
$$

To find out the maximum likelihood estimators of $\alpha, \beta, \sigma, a, b, k, \theta$, we have to solve the above system of nonlinear equations with respect to $\alpha, \beta, \sigma, a, b, k$ and $\theta$ (see appendix).As it seems, this system has no closed form solution in $\alpha, \beta, \sigma, a, b, k, \theta$. Then we have to use a numerical technique method, such as Newton-Raphson method, to obtain the solution.

The approximate confidence intervals of the parameters based on the asymptotic distributions of the MLE of the parameters $\alpha, \beta, \lambda, \gamma, \theta$ are derived in this subsection. For the observed information matrix for $\alpha, \beta, \lambda, \gamma, \theta$, we find the second partial derivatives of $\mathrm{L}$ in appendix. Then the observed information matrix is given by $(\mathbf{I})$

$$
\mathbf{I}=-\left(\begin{array}{ccccccc}
\frac{\partial^{2} L}{\partial \alpha^{2}} & \frac{\partial^{2} L}{\partial \alpha \partial \beta} & \frac{\partial^{2} L}{\partial \alpha \partial \sigma} & \frac{\partial^{2} L}{\partial \alpha \partial a} & \frac{\partial^{2} L}{\partial \alpha \partial b} & \frac{\partial^{2} L}{\partial \alpha \partial k} & \frac{\partial^{2} L}{\partial \alpha \partial \theta} \\
\frac{\partial^{2} L}{\partial \beta \partial \alpha} & \frac{\partial^{2} L}{\partial \beta^{2}} & \frac{\partial^{2} L}{\partial \beta \partial \sigma} & \frac{\partial^{2} L}{\partial \beta \partial a} & \frac{\partial^{2} L}{\partial \beta \partial b} & \frac{\partial^{2} L}{\partial \beta \partial k} & \frac{\partial^{2} L}{\partial \beta \partial \theta} \\
\frac{\partial^{2} L}{\partial \sigma \partial \alpha} & \frac{\partial^{2} L}{\partial \sigma \partial \beta} & \frac{\partial^{2} L}{\partial \sigma^{2}} & \frac{\partial^{2} L}{\partial \sigma \partial a} & \frac{\partial^{2} L}{\partial \sigma \partial b} & \frac{\partial^{2} L}{\partial \sigma \partial k} & \frac{\partial^{2} L}{\partial \sigma \partial \theta} \\
\frac{\partial^{2} L}{\partial a \partial \alpha} & \frac{\partial^{2} L}{\partial a \partial \beta} & \frac{\partial^{2} L}{\partial a \partial \sigma} & \frac{\partial^{2} L}{\partial a^{2}} & \frac{\partial^{2} L}{\partial a \partial b} & \frac{\partial^{2} L}{\partial a \partial k} & \frac{\partial^{2} L}{\partial a \partial \theta} \\
\frac{\partial^{2} L}{\partial b \partial \alpha} & \frac{\partial^{2} L}{\partial b \partial \beta} & \frac{\partial^{2} L}{\partial b \partial \sigma} & \frac{\partial^{2} L}{\partial b \partial a} & \frac{\partial^{2} L}{\partial b^{2}} & \frac{\partial^{2} L}{\partial b \partial k} & \frac{\partial^{2} L}{\partial b \partial \theta} \\
\frac{\partial^{2} L}{\partial k \partial \alpha} & \frac{\partial^{2} L}{\partial k \partial \beta} & \frac{\partial^{2} L}{\partial k \partial \sigma} & \frac{\partial^{2} L}{\partial k \partial a} & \frac{\partial^{2} L}{\partial k \partial b} & \frac{\partial^{2} L}{\partial k^{2}} & \frac{\partial^{2} L}{\partial k \partial \theta} \\
\frac{\partial^{2} L}{\partial \theta \partial \alpha} & \frac{\partial^{2} L}{\partial \theta \partial \beta} & \frac{\partial^{2} L}{\partial \theta \partial \sigma} & \frac{\partial^{2} L}{\partial \theta \partial a} & \frac{\partial^{2} L}{\partial \theta \partial b} & \frac{\partial^{2} L}{\partial \theta \partial k} & \frac{\partial^{2} L}{\partial \theta^{2}}
\end{array}\right)
$$

The variance-covariance matrix may be approximated as $\Sigma=I^{-1}$. Since $\Sigma$ 
involves the parameters $\alpha, \beta, \sigma, a, b, k$ and $\theta$, we replace the parameters by the corresponding MLE's in order to obtain an estimate of $\Sigma$, which is denoted by $\widehat{\Sigma}=\widehat{I}^{-1}$. where $\widehat{I}_{i j}=I_{i j}$ when $(\hat{\alpha}, \hat{\beta}, \hat{\sigma}, \hat{a}, \hat{b}, \hat{k}, \hat{\theta})$ replaces $(\alpha, \beta, \sigma, a, b, k, \theta)$. By using above, approximate $100(1-\delta) \%$ confidence intervals for $\alpha, \beta, \sigma, a, b, k, \theta$ are determined, respectively, as

$$
\begin{gathered}
\widehat{\alpha} \pm z_{\frac{\delta}{2}} \sqrt{\widehat{\Sigma}_{11}} \quad, \quad \widehat{\beta} \pm z_{\frac{\delta}{2}} \sqrt{\widehat{\Sigma}_{22}} \quad, \quad \widehat{\sigma} \pm z_{\frac{\delta}{2}} \sqrt{\widehat{\Sigma}_{33}}, \widehat{a} \pm z_{\frac{\delta}{2}} \sqrt{\widehat{\Sigma}_{44}} \\
\widehat{b} \pm z_{\frac{\delta}{2}} \sqrt{\widehat{\Sigma}_{55}} \quad, \quad \widehat{k} \pm z_{\frac{\delta}{2}} \sqrt{\widehat{\Sigma}_{66}} \text { and } \widehat{\theta} \pm z_{\frac{\delta}{2}} \sqrt{\widehat{\Sigma}_{77}}
\end{gathered}
$$

where $z_{\delta}$ is the upper $\delta$ th percentile of the standard normal distribution.

\section{Simulation Study}

In this section we consider simulation study for the Beta Modified Weibull Poisson (BMWP) distribution, Beta Modified Weibull Geometric (BMWG) distribution, Beta Modified Weibull logarithmic (BMWL) distribution.The comparison is based on mean square error (MSE) for each parameter.

At first, we consider the Beta Modified Weibull Poisson (BMWP) distribution, Beta Modified Weibull Geometric (BMWG) distribution, Beta Modified Weibull logarithmic (BMWL) distribution for data generation for the sample size $n=100$ and 1000 and the true values of the parameters. For estimating of parameters we focus on method of maximizing the full likelihood function.

The likelihood of these four distributions can be maximized by function nlminb in software $\mathbf{R}$. This function may be used for minimization of a function of parameters. For maximization of the likelihood function one may minimize minus log likelihood function. The function nlminb uses optimization method of port routine which is given in http://netlib.bell-labs.com/cm/cs/cstr/153.pdf. The function nlminb uses a sequential quadratic programming (SQP) method to min- imize the requested function. The observed Hessian matrix may be obtained by nlminb function or may be provided by function fdHess.

Table 3 presents the results of the simulation studies for the BMWPS distributions, respectively. The parameter estimates by the model (for $n=50, n=100$ and $n=1000$ ) are close to the true values of the parameters. Of course, the more the value of $n$ the better the estimates. The assessment based on simulation study is that the MSEs for each parameter decreases with increasing the sample size. Figure 6, Figure 7 and Figure 8 show the MSEs for each parameter decreases with increasing the sample size. 
Table 3. Results of the simulation study for the BMWPS distributions. $B=B M W P$, $G=B M W G, L=B M W L, \mathrm{P}=$ Parameter and $\mathrm{T} . \mathrm{v}=$ True value.

\begin{tabular}{|c|c|c|c|c|c|c|c|c|c|c|}
\hline \multirow{2}{*}{$\begin{array}{l}\mathrm{B} \\
\mathrm{P}\end{array}$} & \multirow[b]{2}{*}{ T.v } & \multicolumn{3}{|c|}{$n=50$} & \multicolumn{3}{|c|}{$n=100$} & \multicolumn{3}{|c|}{$n=1000$} \\
\hline & & Est & $\mathrm{SE}$ & MSE & Est & $\mathrm{SE}$ & MSE & Est & $\mathrm{SE}$ & MSE \\
\hline$a$ & 2.000 & 2.132 & 0.410 & 0.185 & 2.122 & 0.343 & 0.133 & 1.953 & 0.113 & 0.015 \\
\hline$b$ & 2.000 & 2.185 & 0.512 & 0.296 & 2.175 & 0.472 & 0.254 & 1.993 & 0.083 & 0.007 \\
\hline$\sigma$ & 1.000 & 0.839 & 0.511 & 0.287 & 0.827 & 0.415 & 0.202 & 1.034 & 0.088 & 0.009 \\
\hline$k$ & 2.000 & 2.142 & 0.423 & 0.199 & 2.138 & 0.311 & 0.116 & 1.930 & 0.010 & 0.005 \\
\hline$\theta$ & 1.000 & 1.459 & 0.211 & 0.255 & 1.363 & 0.191 & 0.168 & 1.072 & 0.029 & 0.006 \\
\hline$\alpha$ & 3.000 & 3.219 & 0.171 & 0.077 & 3.210 & 0.171 & 0.073 & 3.011 & 0.019 & 0.0004 \\
\hline$\beta$ & 3.000 & 3.397 & 0.401 & 0.318 & 3.223 & 0.321 & 0.152 & 3.034 & 0.049 & 0.003 \\
\hline \multicolumn{2}{|l|}{$G$} & \multicolumn{3}{|c|}{$n=50$} & \multicolumn{3}{|c|}{$n=100$} & \multicolumn{3}{|c|}{$n=1000$} \\
\hline $\mathrm{P}$ & T.v & Est & $\mathrm{SE}$ & MSE & Est & $\mathrm{SE}$ & MSE & Est & $\mathrm{SE}$ & MSE \\
\hline$a$ & 2.000 & 2.231 & 0.213 & 0.098 & 2.146 & 0.189 & 0.057 & 1.968 & 0.102 & 0.011 \\
\hline$b$ & 2.000 & 2.241 & 0.231 & 0.111 & 2.215 & 0.228 & 0.098 & 1.988 & 0.109 & 0.012 \\
\hline$\sigma$ & 1.000 & 0.801 & 0.171 & 0.069 & 0.845 & 0.149 & 0.046 & 1.093 & 0.042 & 0.010 \\
\hline$k$ & 2.000 & 2.477 & 0.247 & 0.288 & 2.371 & 0.210 & 0.181 & 1.993 & 0.019 & 0.0004 \\
\hline$\theta$ & 1.000 & 1.448 & 0.239 & 0.258 & 1.440 & 0.234 & 0.168 & 1.081 & 0.035 & 0.007 \\
\hline$\alpha$ & 3.000 & 3.491 & 0.189 & 0.277 & 3.315 & 0.171 & 0.073 & 3.009 & 0.021 & 0.0005 \\
\hline$\beta$ & 3.000 & 3.289 & 0.187 & 0.118 & 3.132 & 0.126 & 0.033 & 3.042 & 0.055 & 0.004 \\
\hline \multicolumn{2}{|l|}{$\mathrm{L}$} & \multicolumn{3}{|c|}{$n=50$} & \multicolumn{3}{|c|}{$n=100$} & \multicolumn{3}{|c|}{$n=1000$} \\
\hline $\mathrm{P}$ & T.v & Est & $\mathrm{SE}$ & MSE & Est & $\mathrm{SE}$ & MSE & Est & $\mathrm{SE}$ & MSE \\
\hline$a$ & 2.000 & 2.372 & 0.210 & 0.182 & 2.321 & 0.142 & 0.123 & 1.997 & 0.011 & 0.0001 \\
\hline$b$ & 2.000 & 2.381 & 0.583 & 0.485 & 2.241 & 0.571 & 0.384 & 1.990 & 0.072 & 0.005 \\
\hline$\sigma$ & 1.000 & 0.901 & 0.550 & 0.312 & 0.910 & 0.532 & 0.291 & 1.006 & 0.004 & 0.00005 \\
\hline$k$ & 2.000 & 2.219 & 0.147 & 0.069 & 2.111 & 0.299 & 0.101 & 1.995 & 0.016 & 0.0002 \\
\hline$\theta$ & 1.000 & 1.159 & 0.189 & 0.061 & 1.109 & 0.142 & 0.032 & 1.009 & 0.007 & 0.0001 \\
\hline$\alpha$ & 3.000 & 3.221 & 0.177 & 0.080 & 3.187 & 0.163 & 0.061 & 3.001 & 0.009 & 0.00008 \\
\hline$\beta$ & 3.000 & 3.280 & 0.280 & 0.131 & 3.112 & 0.267 & 0.083 & 3.005 & 0.009 & 0.00001 \\
\hline
\end{tabular}


Table 4. Lifetimes of 50 devices

\begin{tabular}{rrrrrrrrrrrrrrrrrrr}
\hline \hline 0.1 & 0.2 & 1 & 1 & 1 & 1 & 1 & 2 & 3 & 6 & 7 & 11 & 12 & 18 & 18 & 18 & 18 & 18 & 21 \\
32 & 36 & 40 & 45 & 46 & 47 & 50 & 55 & 60 & 63 & 63 & 67 & 67 & 67 & 67 & 72 & 75 & 79 & 82 \\
82 & 83 & 84 & 84 & 84 & 85 & 85 & 85 & 85 & 85 & 86 & 86 & & & & & & & \\
\hline
\end{tabular}

\section{Application}

From the current study, it is hoped that BMWPS distribution can be used more widely in both a theoretical and an applicable aspect. In this section, we analyze two real data sets to demonstrate the performance of the BMWPS distribution in practice. One of the data sets is a sample of 50 components taken from Aarset (1987).

We illustrate the superiority of the new distribution as compared to some of its sub-models. We then perform the goodness of fit of the BMWL distribution and submodels, which allow their evaluation relative to each other and to the more general BMWL model. However, the lower values of AIC and BIC for the BMWL and other distributions indicate that these models could be chosen as the best models to fit the data. In addition to comparing the models, we use two other criteria. First, we consider the LR statistic and next we consider formal goodness-of-fit tests. The required numerical evaluations are implemented using the $\mathrm{R}$ softwares.

\section{The Aarset Data}

We consider the widely used data from Aarset (1987), and also reported in Mudholkar and Srivastava (1993), Mudholkar et al. (1996) and Wang (2000), on lifetimes of 50 components, which possess a bathtub-shaped failure rate property. The data contains the times to failure of 50 devices put on life test at time 0 , from Magne Vollan Aarset (1987) as in Table 4.

Table 5 lists the MLEs of the model parameters and the following statistics: Akaike information criterion (AIC), Bayesian information criterion (BIC) and -2 $\log$-likelihood $(-2 \log (L))$. These results show that the BMW distribution has the lowest $\mathrm{AIC}, \mathrm{BIC}$ and $-2 \log (L)$ values among all the fitted models, and so it could be chosen as the best model.

We can compute the maximum values of the unrestricted and restricted loglikelihoods to obtain the LR statistics for testing some sub-models of the BMWPS distribution. For example, the test of the BMW distribution against the BMWL model can be described as the test of $H_{0}: \theta \downarrow_{0}$ versus $H_{1}: \theta \downarrow_{0} \quad(\theta>0)$. Generally, we consider the partition $\Theta=\left(\Theta_{1}, \Theta_{2}\right)$ of the parameter of the BMWPS distribution, where $\Theta_{1}$ is a subset of parameters of interest and $\Theta_{2}$ is a subset of the remaining parameters. Let, $\widehat{\Theta}$ and $\widetilde{\Theta}$ be the MLEs under the alternative and null hypotheses, respectively, and $\Theta_{1}^{0}$ a specified parameter vector. Then, the LR statis- 
Table 5. MLEs of the model parameters, and the measures of AIC ,BIC and $-2 \log (L)$ for Aarset data (values of best fitted model are highlighted).

\begin{tabular}{llllllll}
\hline \hline & \multicolumn{7}{c}{ Estimations } \\
\cline { 2 - 7 } Distribution & $\hat{\alpha}$ & $\hat{\beta}$ & $\hat{\sigma}$ & $\hat{a}$ & $\hat{b}$ & $\hat{k}$ & $\hat{\theta}$ \\
\hline BMWP & 0.0193 & 0.2202 & 0.1859 & 1.695 & 1.0447 & 1.0009 & $8.70 \mathrm{E}-09$ \\
BMWG & 0.0081 & & 0.1024 & 1.0639 & 0.9733 & 0.153 & \\
BMWL & 0.0083 & 0.082 & 1.2893 & 1.2175 & 0.2468 & $9.00 \mathrm{E}-10$ \\
MWP & 0.016 & & 0.2384 & & & 0.6197 & $1.58 \mathrm{E}-08$ \\
MWG & 0.0192 & & 0.0972 & & & 0.8176 & 0 \\
MWL & 0.0187 & & 0.298 & & & 0.7798 & $1.17 \mathrm{E}-08$ \\
WP & 0.0075 & & 1.1726 & & & 0.5847 & $4.00 \mathrm{E}-10$ \\
WG & 0.0022 & & 1.4022 & & & 0.4284 & 0 \\
WL & 0.2407 & & 0.5455 & & & 1.923 & $1.19 \mathrm{E}-08$ \\
BMW & & $5.54 \mathrm{E}-07$ & 3.8881 & 0.0104 & 0.1077 & 10.5054 & \\
MW & 0.0186 & 0.0404 & 0.3735 & & & & \\
W & 0.027 & & 0.949 & & & & \\
\hline
\end{tabular}

\begin{tabular}{lrrr}
\hline \hline & \multicolumn{3}{c}{ Statistic } \\
\cline { 2 - 4 } Distribution & AIC & BIC & $-2 \log (L)$ \\
& & & \\
\hline BMWP & 490.73 & 504.11 & 476.73 \\
BMWG & 553.82 & 567.2 & 539.82 \\
BMWL & 515.86 & 529.24 & 501.86 \\
MWP & 491.66 & 501.22 & 481.66 \\
MWG & 490.07 & 499.63 & 480.07 \\
MWL & 489.99 & 499.55 & 479.99 \\
WP & 485.69 & 493.34 & 477.69 \\
WG & 483.89 & 491.53 & 475.89 \\
WL & 501.24 & 508.89 & 493.24 \\
BMW & 453.27 & 464.74 & 441.27 \\
MW & 484.97 & 490.7 & 478.97 \\
W & 486.00 & 489.83 & 482.00 \\
\hline
\end{tabular}


Table 6. LR tests for Aarset data.

\begin{tabular}{lcc}
\hline \hline Model & LR-statistics & $p$-value \\
\hline MWP vs. BMWP & 4.92882 & 0.08506 \\
BMW vs. BMWP & 35.45762 & 0.00000 \\
W vs. BMWP & 5.27382 & 0.08338 \\
WG vs. BMWG & 63.93106 & 0.00000 \\
MW vs. BMWG & 60.84836 & 0.00000 \\
MWL vs. BMWL & 21.86915 & 0.00000 \\
BMW vs. BMWL & 60.58725 & 0.00000 \\
W vs. BMWL & 19.85581 & 0.00000 \\
WP vs. BMWP & 0.96453 & 0.01901 \\
MW vs. BMWP & 2.23859 & 0.00803 \\
MWG vs. BMWG & 59.75063 & 0.00000 \\
BMW vs. BMWG & 98.54457 & 0.00000 \\
W vs. BMWG & 57.81313 & 0.00000 \\
WL vs. BMWL & 8.6204 & 0.00000 \\
MW vs. BMWL & 22.89104 & 0.00000 \\
& & \\
\hline
\end{tabular}

tic for testing the null hypothesis $H_{0}: \Theta_{1}=\Theta_{1}^{0}$ against the alternative hypothesis $H_{1}: \Theta_{1} \neq \Theta_{1}^{0}$. Also, the values of some more LR statistics are given in Table 6 . Therefore, under the null hypothesis of $\theta \downarrow_{0}$, LR statistics (test statistic) is 60.58725 and then the $p$-value $=0.00000$. Since the $\mathrm{p}$-value is less than $1 \%$, we are very confident to reject our null hypothesis, i.e., According to Tables 5 and 6 , the model BMW is the best for the device failure data. Also, some other values of LR statistics are given in Table 6 .

Now, We consider the Cramer-von Mises $\left(W^{*}\right)$ and Anderson-Darling $\left(A^{*}\right)$ statistics. In general, the smaller the values of the statistics $W^{*}$ and $A^{*}$, the better the fit to the data. The statistics $W^{*}$ and $A^{*}$ are described in detail in Chen and Balakrishnan (1995). The values of the statistics $W^{*}$ and $A^{*}$ for all models are given in Table 7.

Based on these statistics (in Table 7), we conclude that the BMWPS models fits the current data better than the other models. As mentioned before, Figure 9, shows that the BMWL distribution could be chosen as the best model because it does fit better to the survival function of these data than the other sub-models. The TTT plot for these data, in Figure 10, shows an bathtub-shaped hazard rate function and, therefore, indicates the appropriateness of the BMWL distribution to fit these data. 
Table 7. Goodness-of-fit tests for Aarset data.

\begin{tabular}{ccccc}
\hline \hline & \multicolumn{4}{c}{ Statistic } \\
\cline { 2 - 5 } Distribution & $A^{*}$ & $W^{*}$ & K-S & $p$-value \\
\hline BMWP & 2.37385 & 0.39926 & 0.17215 & 0.10324 \\
BMWL & 8.42736 & 1.66036 & 0.3031 & 0.0002 \\
MWG & 5.60459 & 0.55402 & 0.27316 & 0.00115 \\
WP & 3.33473 & 0.60581 & 0.21064 & 0.02367 \\
WL & 37.17469 & 1.28354 & 0.54368 & 0.00000 \\
MW & 2.93647 & 0.51482 & 0.1941 & 0.04621 \\
BMWG & 22.66601 & 4.53222 & 0.46073 & 0.00000 \\
MWP & 3.64417 & 0.69114 & 0.22252 & 0.01414 \\
MWL & 5.21034 & 0.57114 & 0.26503 & 0.00178 \\
WG & 2.71079 & 0.67182 & 0.1925 & 0.04918 \\
BMW & 0.86983 & 0.09965 & 0.12062 & 0.46092 \\
W & 3.54445 & 0.53486 & 0.1928 & 0.04860 \\
\hline
\end{tabular}

\section{Conclusion}

We introduce a seven parameter lifetime distribution called "A Beta Modified Weibull Power Series (BMWPS) Distribution" which is a new mixed distribution of the Beta modified Weibull and Power Series distribution. The BMWPS model studied in this paper is flexible in modeling various types of failure data with possibly bathtub shaped failure rate function. Furthermore, we provide a mathematical treatment of this distribution including the order statistics. Also, we provide explicit expressions for the density function of the order statistics and their moments. Several properties of the BMWPS distribution such as quantiles and moments, maximum likelihood are studied. Finally, we fitted BMWPS model to one real data sets to show the potential of the new proposed distribution. We hope that the proposed extended model may attract wider applications in survival analysis, the literature of the exciting life distributions and reliability engineers.

\section{References}

Aarset, M.V. (1987). How to Identify Bathtub Hazard Rate. IEEE Transactions on Reliability, 36, 106-108.

Bagheri, S.F., Samani, E.B. and Ganjali, M. (2016). The Generalized Modified Weibull Power Series Distribution: Theory and Applications. Computational Statistics and Data Analysis, 94, 136-160. 
Bebbington, M., Lai, C.D. and Zitikis, R. (2007). A Flexible Weibull Extension. Reliability Engineering and System Safety, 92, 719-726.

Barlow, R.E., Campo, R. (1975). Total Time on Test Processes and Applications to Failure Data Analysis. In: Reliability and Fault Tree Analysis. Society for Industrial and Applied Mathematics, 451-481.

Carrasco, J.M.F., Ortega, E.M.M. and Cordeiro, G.M. (2008). A Generalized Modified Weibull Distribution for Lifetime Modeling. Computational Statistics and Data Analysis: doi 10.1016/j.CSDA.2008.08.023.

Cordeiro, G.M., Simas, A.B. and Stoesiac, B.D. (2008). The Beta Weibull Distribution. Submitted to Journal of Statistical Computation and Simulation.

Glaser, R.E. (1980). Bathtub and Related Failure Rate Characterizations. Journal of the American Statistical Association, 75, 667-672.

Haupt, E. and Schabe, H. (1992). A New Model for a Lifetime Distribution with Bathtub Shaped Failure Rate. Microelect. and Reliab, 32, 633-639.

Hjorth, U. (1980). A Realibility Distribution with Increasing, Decreasing, Constant and Bathtub Failure Rates. Technometrics, 22, 99-107.

Lai, C.D., Xie, M. and Murthy, D.N.P. (2003). A Modified Weibull Distribution. Transactions on Reliability, 52, 33-37.

Mudholkar, G.S. and Srivastava, D.K. (1993). Exponentiated Weibull Family for Analyzing Bathtub Failure-real Data. IEEE Trans Reliab, 42, 299-302.

Mudholkar, G.S., Srivastava, D.K. and Friemer, M. (1995). The Exponentiated Weibull Family: A Reanalysis of the Bus-motor-failure Data. Technometrics, 37, 436-445.

Mudholkar, G.S., Srivastava, D.K. and Kollia, G.D. (1996). A Generalization of the Weibull Distribution with Application to the Analysis of Survival Data. J. Amer. Statist. Assoc., 91, 1575-1583.

Nadarajah, S. and Kotz, S. (2004). The Beta Gumbel Distribution. Mathematical Problems in Engineering, 323-332.

Nelson, W. (1982). Lifetime Data Analysis. Wiley, New York.

Pham, H. and Lai, C.D. (2007). On Recent Generalizations of the Weibull Distribution. IEEE Transactions on Reliability, 56, 454-458.

Rajarshi, S. and Rajarshi, M.B. (1988). Bathtub Distributions: a Review. Comm. Statat. Theory. Meth., 17, 2521-2597. 
Silva, G.O., Ortega, E.M. and Cordeiro, G.M., (2010). The Beta Modified Weibull Distribution. Lifetime Data Analysis, 16, 409-430.

Wang, F.K. (2000). A New Model with Bathtub-shaped Failure Rate Using an Additive Burr XII Distribution. Reliability Engineering and System Safety, 70, 305-312.

Xie, M. and Lai, C.D. (1995). Reliability Analysis Using an Additive Weibull Model with Bathtub Shaped Failure Rate Function. Reliab. Eng. Syst. Safety, 52, 87-93.

Xie, M., Tang, Y. and Goh, T.N. (2002). A Modified Weibull Extension with Bathtub Failure Rate Function. Reliab. Eng. Syst. Safety, 76, 279-285. 


\section{Appendix}

For calculating the maximum likelihood function, we determine the first partial derivatives of $L$ with respect to $\alpha, \beta, \sigma, a, b, k, \theta$ and equal them to zero as following.

$$
\begin{aligned}
& \frac{\partial L}{\partial \alpha}=(a-1) \sum_{i=1}^{n} \frac{x_{i} e^{-\alpha x_{i}-\beta x_{i}^{\sigma}}}{1-e^{-\alpha x_{i}-\beta x_{i}^{\sigma}}}+(b-1) \sum_{0}^{n} \frac{-x_{i} e^{-\alpha x_{i}-\beta x_{i}^{\sigma}}}{e^{-\alpha x_{i}-\beta x_{i}^{\sigma}}}+\frac{n}{\alpha}-\sum_{i=1}^{n} x_{i} \\
& +(k-1) \sum_{i=1}^{n} \frac{\left(1-e^{-\alpha x_{i}-\beta x_{i}^{\sigma}}\right)^{a-1}\left(e^{-\alpha x_{i}-\beta x_{i}^{\sigma}}\right)^{b-1} x_{i} e^{-\alpha x_{i}-\beta x_{i}^{\sigma}}}{\int_{0}^{1-e^{-\alpha x_{i}-\beta x_{i}^{\sigma}}} t^{a-1}(1-t)^{b-1} d t} \\
& +\theta k \sum_{i=1}^{n}\left\{\frac{C^{\prime \prime}\left(\theta\left(1-\left(I_{1-e^{-\alpha x_{i}-\beta x_{i}^{\sigma}}}(a, b)\right)^{k}\right)\right)}{C^{\prime}\left(\theta\left(1-\left(I_{1-e^{-\alpha x_{i}-\beta x_{i}^{\sigma}}}(a, b)\right)^{k}\right)\right)}\left(I_{1-e^{-\alpha x_{i}-\beta x_{i}^{\sigma}}}(a, b)\right)^{k-1}\right. \\
& \left.\times\left(1-e^{-\alpha x_{i}-\beta x_{i}^{\sigma}}\right)^{a-1}\left(e^{-\alpha x_{i}-\beta x_{i}^{\sigma}}\right)^{b-1} x_{i} e^{-\alpha x_{i}-\beta x_{i}^{\sigma}}\right\}
\end{aligned}
$$

$$
\begin{aligned}
& \frac{\partial L}{\partial \beta}=(a-1) \sum_{i=1}^{n} \frac{x_{i}^{\sigma} e^{-\alpha x_{i}-\beta x_{i}^{\sigma}}}{1-e^{-\alpha x_{i}-\beta x_{i}^{\sigma}}}+(b-1) \sum_{0}^{n} \frac{-x_{i}^{\sigma} e^{-\alpha x_{i}-\beta x_{i}^{\sigma}}}{e^{-\alpha x_{i}-\beta x_{i}^{\sigma}}}+\sum_{i=1}^{n} \frac{\sigma x_{i}^{\sigma-1}}{\alpha+\beta \sigma x_{i}^{\sigma-1}} \\
& +\sum_{i=1}^{n} x_{i}^{\sigma}+(k-1) \sum_{i=1}^{n} \frac{\left(1-e^{-\alpha x_{i}-\beta x_{i}^{\sigma}}\right)^{a-1}\left(e^{-\alpha x_{i}-\beta x_{i}^{\sigma}}\right)^{b-1} x_{i}^{\sigma} e^{-\alpha x_{i}-\beta x_{i}^{\sigma}}}{\int_{0}^{1-e^{-\alpha x_{i}-\beta x_{i}^{\sigma}}} t^{a-1}(1-t)^{b-1} d t} \\
& +\theta k \sum_{i=1}^{n}\left\{\frac{C^{\prime \prime}\left(\theta \left(1-\left(I_{\left.\left.1-e^{-\alpha x_{i}-\beta x_{i}^{\sigma}}(a, b)\right)^{k}\right)}\right)\right.\right.}{C^{\prime}\left(\theta\left(1-\left(I_{1-e^{-\alpha x_{i}-\beta x_{i}^{\sigma}}}(a, b)\right)^{k}\right)\right)}\left(I_{1-e^{-\alpha x_{i}-\beta x_{i}^{\sigma}}}(a, b)\right)^{k-1}\right. \\
& \left.\times\left(1-e^{-\alpha x_{i}-\beta x_{i}^{\sigma}}\right)^{a-1}\left(e^{-\alpha x_{i}-\beta x_{i}^{\sigma}}\right)^{b-1} x_{i}^{\sigma} e^{-\alpha x_{i}-\beta x_{i}^{\sigma}}\right\}
\end{aligned}
$$




$$
\begin{aligned}
& \frac{\partial L}{\partial \sigma}=(a-1) \sum_{i=1}^{n} \frac{\beta x_{i}^{\sigma} \ln \left(x_{i}\right) e^{-\alpha x_{i}-\beta x_{i}^{\sigma}}}{1-e^{-\alpha x_{i}-\beta x_{i}^{\sigma}}}+(b-1) \sum_{0}^{n} \frac{-\beta x_{i}^{\sigma} \ln \left(x_{i}\right) e^{-\alpha x_{i}-\beta x_{i}^{\sigma}}}{e^{-\alpha x_{i}-\beta x_{i}^{\sigma}}} \\
& +\sum_{i=1}^{n} \frac{\beta x_{i}^{\sigma-1}+\beta \sigma x_{i}^{\sigma-1} \ln \left(x_{i}\right)}{\alpha+\beta \sigma x_{i}^{\sigma-1}}+\sum_{i=1}^{n}-\beta x_{i}^{\sigma} \ln \left(x_{i}\right) \\
& +(k-1) \sum_{i=1}^{n} \frac{\left(1-e^{-\alpha x_{i}-\beta x_{i}^{\sigma}}\right)^{a-1}\left(e^{-\alpha x_{i}-\beta x_{i}^{\sigma}}\right)^{b-1} \beta x_{i}^{\sigma} \ln \left(x_{i}\right) e^{-\alpha x_{i}-\beta x_{i}^{\sigma}}}{\int_{0}^{1-e^{-\alpha x_{i}-\beta x_{i}^{\sigma}}} t^{a-1}(1-t)^{b-1} d t} \\
& +\theta k \sum_{i=1}^{n}\left\{\frac{C^{\prime \prime}\left(\theta\left(1-\left(I_{1-e^{-\alpha x_{i}-\beta x_{i}^{\sigma}}}(a, b)\right)^{k}\right)\right)}{C^{\prime}\left(\theta\left(1-\left(I_{1-e^{-\alpha x_{i}-\beta x_{i}^{\sigma}}}(a, b)\right)^{k}\right)\right)}\left(I_{1-e^{-\alpha x_{i}-\beta x_{i}^{\sigma}}}(a, b)\right)^{k-1}\right. \\
& \left.\times\left(1-e^{-\alpha x_{i}-\beta x_{i}^{\sigma}}\right)^{a-1}\left(e^{-\alpha x_{i}-\beta x_{i}^{\sigma}}\right)^{b-1} \beta x_{i}^{\sigma} \ln \left(x_{i}\right) e^{-\alpha x_{i}-\beta x_{i}^{\sigma}}\right\} \\
& \frac{\partial L}{\partial a}=\sum_{i=1}^{n} \ln \left(1-e^{-\alpha x_{i}-\beta x_{i}^{\sigma}}\right) \\
& +\sum_{i=1}^{n}\left\{\frac{D(k-1)}{\int_{0}^{\left(1-e^{-\alpha x_{i}-\beta x_{i}^{\sigma}}\right.} t^{a-1} d t}+\frac{E(k-1)}{\int_{0}^{\left(1-e^{-\alpha x_{i}-\beta x_{i}^{\sigma}}\right.} t^{a} d t}+\frac{F(k-1)}{\int_{0}^{\left(1-e^{-\alpha x_{i}-\beta x_{i}^{\sigma}}\right.} t^{a+1} d t}\right\} \\
& +\theta k \sum_{i=1}^{n}\left\{\frac{C^{\prime \prime}\left(\theta\left(1-\left(I_{1-e^{-\alpha x_{i}-\beta x_{i}^{\sigma}}}(a, b)\right)^{k}\right)\right)}{C^{\prime}\left(\theta\left(1-\left(I_{1-e^{-\alpha x_{i}-\beta x_{i}^{\sigma}}}(a, b)\right)^{k}\right)\right)}\left(I_{1-e^{-\alpha x_{i}-\beta x_{i}^{\sigma}}}(a, b)\right)^{k-1}\right. \\
& \left.\times\left(\frac{M}{B(a, b)}+\frac{\Gamma(a+b)\left(\psi^{(0)}(a+b)-\psi^{(0)}(a)\right)}{\Gamma(a) \Gamma(b)} \int_{0}^{1-e^{-\alpha x_{i}-\beta x_{i}^{\sigma}}} t^{a-1}(1-t)^{b-1} d t\right)\right\}
\end{aligned}
$$

where

$$
\begin{gathered}
D=\frac{\left(1-e^{-\alpha x_{i}-\beta x_{i}^{\sigma}}\right)^{a}\left(a \ln \left(1-e^{-\alpha x_{i}-\beta x_{i}^{\sigma}}\right)-1\right)}{a^{2}}, \\
E=\frac{(1-b)\left(1-e^{-\alpha x_{i}-\beta x_{i}^{\sigma}}\right)^{a+1}\left((a+2) \ln \left(1-e^{-\alpha x_{i}-\beta x_{i}^{\sigma}}\right)-1\right)}{(a+1)^{2}}, \\
F=\frac{(b-1)(b-2)\left(1-e^{-\alpha x_{i}-\beta x_{i}^{\sigma}}\right)^{a+2}\left((a+2) \ln \left(1-e^{-\alpha x_{i}-\beta x_{i}^{\sigma}}\right)-1\right)}{2(a+2)^{2}},
\end{gathered}
$$




$$
M=\frac{D}{\int_{0}^{\left(1-e^{-\alpha x_{i}-\beta x_{i}^{\sigma}}\right.} t^{a-1} d t}+\frac{E}{\int_{0}^{\left(1-e^{-\alpha x_{i}-\beta x_{i}^{\sigma}}\right.} t^{a} d t}+\frac{F}{\int_{0}^{\left(1-e^{-\alpha x_{i}-\beta x_{i}^{\sigma}}\right.} t^{a+1} d t}
$$

and $\psi^{(n)}(x)$ is the $n$th derivative of the digamma function.

$$
\begin{aligned}
& \frac{\partial L}{\partial b}=\sum_{i=1}^{n} \ln \left(e^{-\alpha x_{i}-\beta x_{i}^{\sigma}}\right)+(k-1) \frac{\partial L}{\partial b} \sum_{i=1}^{n} \ln \left(\int_{0}^{1-e^{-\alpha x_{i}-\beta x_{i}^{\sigma}}} t^{a-1}(1-t)^{b-1} d t\right) \\
& +\theta k \sum_{i=1}^{n}\left\{\frac{C^{\prime \prime}\left(\theta \left(1-\left(I _ { 1 - e ^ { - \alpha x _ { i } - \beta x _ { i } ^ { \sigma } } ( a , b ) ) ^ { k } ) ) } C ^ { \prime } \left(\theta \left(1-\left(I_{\left.\left.\left.1-e^{-\alpha x_{i}-\beta x_{i}^{\sigma}}(a, b)\right)^{k}\right)\right)}\right)\right.\right.\right.\right.\right.}{\left.I_{1-e^{-\alpha x_{i}-\beta x_{i}^{\sigma}}}(a, b)\right)^{k-1}}\right. \\
& \left.\quad \times\left(\frac{1}{B(a, b)} N+\frac{\Gamma(a+b)\left(\psi^{(0)}(a+b)-\psi^{(0)}(b)\right)}{\Gamma(a) \Gamma(b)} \int_{0}^{1-e^{-\alpha x_{i}-\beta x_{i}^{\sigma}}} t^{a-1}(1-t)^{b-1} d t\right)\right\}
\end{aligned}
$$

where

$$
\begin{aligned}
& N=\frac{\partial L}{\partial b} \ln \left(\int_{0}^{1-e^{-\alpha x_{i}-\beta x_{i}^{\sigma}}} t^{a-1}(1-t)^{b-1} d t\right) \\
& \frac{\partial L}{\partial k}=\frac{n}{k}+n \ln \frac{1}{B(a, b)}+\sum_{i=1}^{n} \ln \left(\int_{0}^{1-e^{-\alpha x_{i}-\beta x_{i}^{\sigma}}} t^{a-1}(1-t)^{b-1} d t\right) \\
& +\theta \sum_{i=1}^{n}\left\{\frac{C^{\prime \prime}\left(\theta\left(1-\left(I_{1-e^{-\alpha x_{i}-\beta x_{i}^{\sigma}}}(a, b)\right)^{k}\right)\right)}{C^{\prime}\left(\theta\left(1-\left(I_{1-e^{-\alpha x_{i}-\beta x_{i}^{\sigma}}}(a, b)\right)^{k}\right)\right)}\right. \\
& \times\left(\frac{1}{B(a, b)} \int_{0}^{1-e^{-\alpha x_{i}-\beta x_{i}^{\sigma}}} t^{a-1}(1-t)^{b-1} d t\right)^{k} \\
& \left.\times \ln \left(\frac{1}{B(a, b)} \int_{0}^{1-e^{-\alpha x_{i}-\beta x_{i}^{\sigma}}} t^{a-1}(1-t)^{b-1} d t\right)\right\} \\
& \frac{\partial L}{\partial \theta}=\frac{n}{\theta}-\frac{n C^{\prime \prime}(\theta)}{C^{\prime}(\theta)}+\sum_{i=1}^{n}\left\{\frac{C^{\prime \prime}\left(\theta\left(1-\left(I_{1-e^{-\alpha x_{i}-\beta x_{i}^{\sigma}}}(a, b)\right)^{k}\right)\right)}{C^{\prime}\left(\theta\left(1-\left(I_{1-e^{-\alpha x_{i}-\beta x_{i}^{\sigma}}}(a, b)\right)^{k}\right)\right)}\right. \\
& \left.\times\left(1-\left(\frac{1}{B(a, b)} \int_{0}^{1-e^{-\alpha x_{i}-\beta x_{i}^{\sigma}}} t^{a-1}(1-t)^{b-1} d t\right)^{k}\right)\right\} .
\end{aligned}
$$



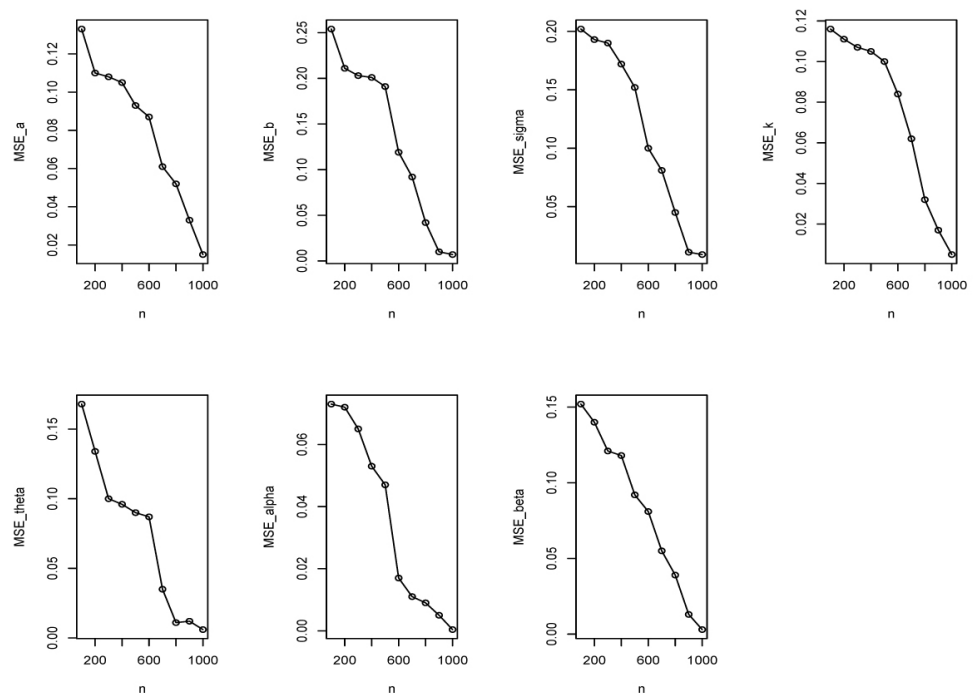

Figure 6. Mean squared errors of the MLEs (BMWG) of each parameter versus $n$
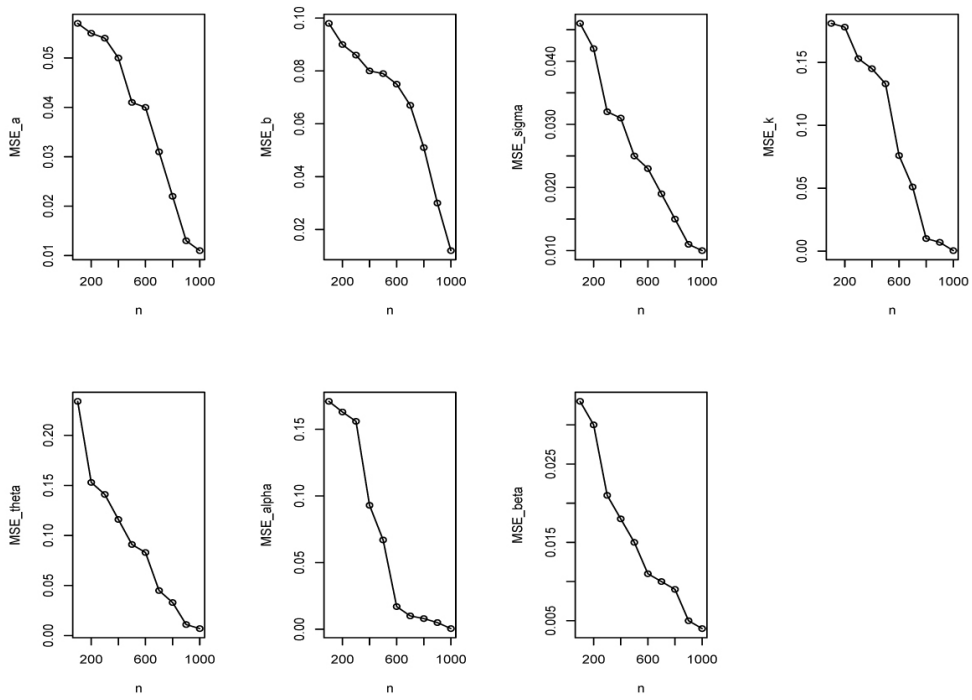

Figure 7. Mean squared errors of the MLEs (BMWG) of each parameter versus $n$ 

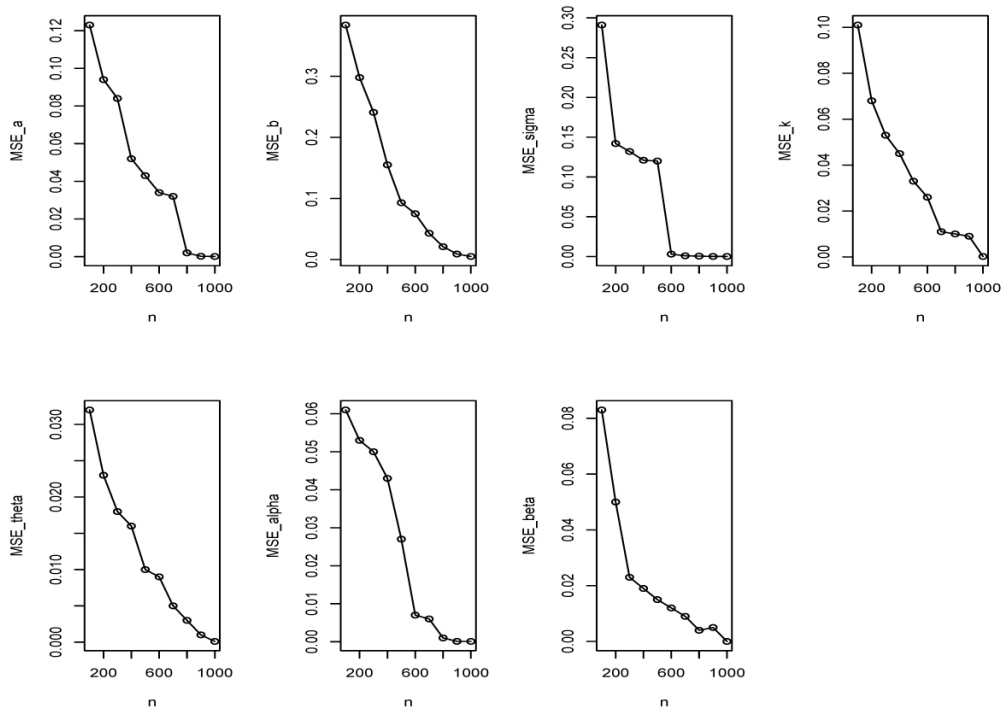

Figure 8. Mean squared errors of the MLEs (BMWL) of each parameter versus $n$
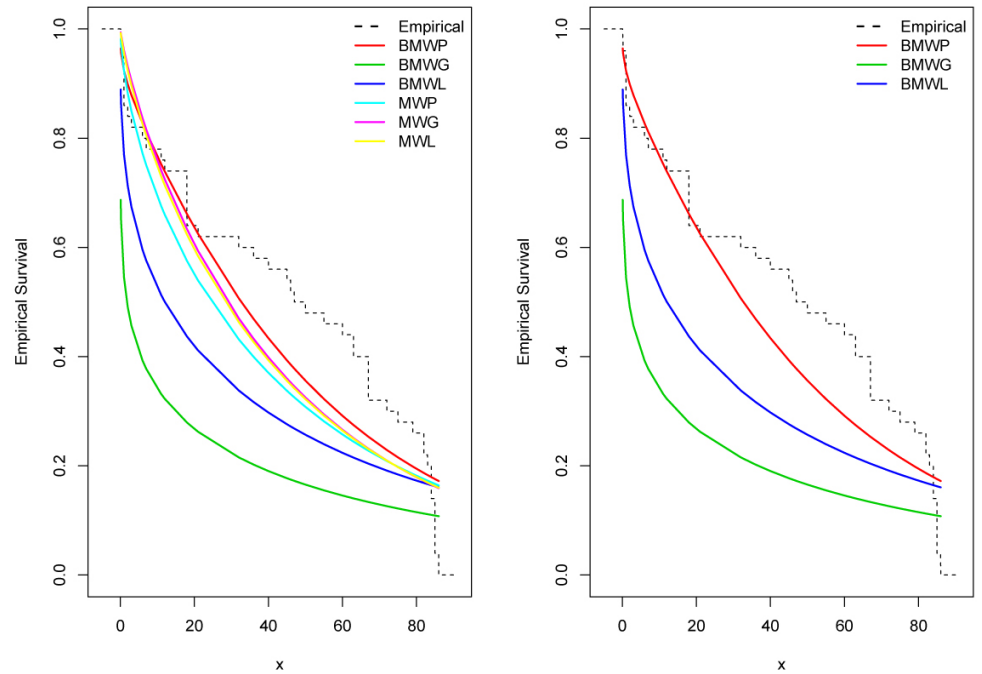

Figure 9. Estimated survival function and the empirical survival for Aarset data. 

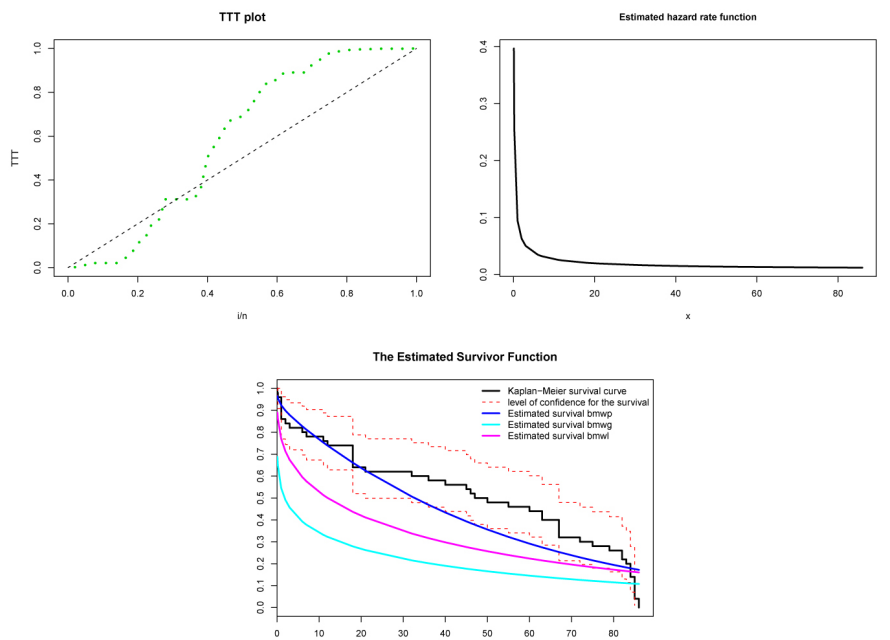

Figure 10. Left panels: empirical TTT-plot. Right panels: Estimated hazard rate function. Bottom panels: Estimated survival functions for three fitted models and the empirical survival function. All for Aarset data.

\section{Narges Yarmoghaddam}

Department of Statistics,

Shahid Beheshti University,

Tehran, Iran.

email: n_yarmoghaddam@yahoo.com

\section{Ehsan Bahrami Samani}

Department of Statistics, Shahid Beheshti University,

Tehran, Iran.

email: ehsan_bahrami_samani@yahoo.com 
